Table . Comparison of patients undergoing 2nd RB according to development of renal failure

\begin{tabular}{|c|c|c|c|}
\hline & Renal failure $(n=26)$ & $\begin{array}{l}\text { No renal failure } \\
\qquad(\mathrm{n}=69)^{*}\end{array}$ & $\mathrm{p}$ \\
\hline Total FU (years), mean (SD) & $21(10.4)$ & $16.5(9.39)$ & 0.002 \\
\hline $\mathrm{SCr}(\mathrm{mg} / \mathrm{dl})$ at $2^{\circ} \mathrm{RB}$, mean (SD) & $1.7(1)$ & $0.98(0.35)$ & 0.001 \\
\hline Proteinuria $(\mathrm{g} / 24 \mathrm{~h})$ at $2^{\circ} \mathrm{RB}$, mean (SD) & $4.7(3.9)$ & $2.99(2.63)$ & 0.022 \\
\hline Class IV and IV+V at $2^{\circ} \mathrm{RB}, \%$ & 76.9 & 54.5 & 0.07 \\
\hline Hypertension at onset, \% & 84.6 & 32.4 & $<0.001$ \\
\hline $\mathrm{HCQ}$ intake at $2^{\circ} \mathrm{RB}, \%$ & 9.5 & 52 & $<0.001$ \\
\hline Glucocorticoids at $2^{\circ} \mathrm{RB} \%$ & 84 & 87 & ns \\
\hline Immunosuppressants at $2^{\circ} \mathrm{RB} \%$ & 40 & 58 & ns \\
\hline $\mathrm{Al}$ at onset, mean (SD) & $7.14(3.95)$ & $7.02(3.86)$ & ns \\
\hline Al at $2^{\circ} \mathrm{RB}$, mean (SD) & $5.37(4.12)$ & $4.02(3.71)$ & ns \\
\hline $\mathrm{Cl}$ at onset, mean (SD) & $2.05(1.88)$ & $1.56(1.64)$ & ns \\
\hline $\mathrm{Cl}$ at $2^{\circ} \mathrm{RB}$, mean (SD) & $3.87(3.08)$ & $3.52(2.16)$ & ns \\
\hline Years between $2^{\circ} \mathrm{RB}$ and end of FU, mean (SD) & $14.1(10.5)$ & $9.3(8.84)$ & ns \\
\hline
\end{tabular}

*Group 1 excluded

$\mathrm{RB}$, renal biopsy; $\mathrm{Al}$, activity index; $\mathrm{Cl}$, chronicity index; $\mathrm{SCr}$ serum creatinine; $\mathrm{FU}$, follow-up; $\mathrm{SD}$, standard deviation.

Disclosure of Interests: Mariele Gatto Speakers bureau: GSK, Francesca Saccon: None declared, Francesca Radice: None declared, Paolo Gilles Vercelloni: None declared, Renato Alberto Sinico: None declared, Giulia Frontini: None declared, Valentina Binda: None declared, Piergiorgio Messa: None declared, Federico Alberici: None declared, Gabriella Moroni: None declared, Andrea Doria Consultant of: GSK, Pfizer, Abbvie, Novartis, Ely Lilly, Speakers bureau: UCB pharma, GSK, Pfizer, Janssen, Abbvie, Novartis, Ely Lilly, BMS DOI: 10.1136/annrheumdis-2020-eular.3523 LUPUS NEPHRITIS AND RESPONSE TO TREATMENT

J. Davies ${ }^{1}$, A. Midgley ${ }^{1}$, S. Donohue ${ }^{1}$, I. N. Bruce ${ }^{2}$, M. Beresford ${ }^{1}, \underline{\text { C. Hedrich }}{ }^{1}$ on behalf of the MRC MASTERPLANS and BILAG-BR Consortia. ${ }^{1}$ University of Liverpool, Institute of Translational Medicine, Department of Women's and Children's Health, Liverpool, United Kingdom; ${ }^{2}$ University of Manchester, Arthritis Research UK Epidemiology Unit, Faculty of Biology Medicine and Health, Manchester, United Kingdom

Background: Systemic lupus erythematosus (SLE) is a systemic autoimmune/ inflammatory disease. Approximately $30 \%$ of SLE patients develop lupus nephritis (LN) that affects treatment and prognosis. Easily accessible biomarkers do not exist to reliably predict renal disease ${ }^{1}$. Recently, calcium-binding $\mathrm{S} 100$ proteins have been suggested as biomarkers in systemic inflammatory conditions, including SLE ${ }^{2,3}$

Objectives: The MASTERPLANS Consortium aims to identify indicators of treatment responses in SLE. This study tested the applicability of S100 proteins in serum and urine as biomarkers for disease activity and response to treatment with rituximab in $\mathrm{LN}$.

Methods: S100A8/A9 and S100A12 proteins were quantified in the serum and urine of 243 SLE patients from the BILAG-BR study and 48 matched controls using MSD technology to determine whether they perform as biomarkers for active $\mathrm{LN}$ ( $\mathrm{n}=85$ SLE patients) and/or may be used to predict response to treatment with rituximab. Renal disease activity and response to treatment was based on BILAG-BR scores and changes in response to treatment ${ }^{4,5}$

Results: Serum S100A12 ( $<<0.001)$, and serum and urine S100A8/A9 $(p<0.001)$ are elevated in SLE patients. While serum and urine $\mathrm{S} 100$ levels do not correlate with global SLE disease activity (SLEDAI), levels in urine and urine/serum ratios are elevated in SLE patients with active LN (S100A8/A9: urine $p>0.005$, urine/ serum $p<0.05$; $S 100 A 12$ : urine $p<0.05$, serum/urine $p<0.005)$. S100 proteins perform better as biomarkers for active LN involvement in SLE patients positive for anti-dsDNA antibodies. Lastly, binary logistic regression and AUC analysis suggests the combination of serum S100A8/A9 and S100A12 to predict response to RTX treatment in LN after 6 months.

Conclusion: Findings from this study show promise for clinical application of S100 proteins to predict active renal disease in SLE and response to treatment with rituximab. Significantly overlapping values between groups currently prohibit the definition of cut-off values and prospective studies are required to validate findings.

References:

[1] Reppe Moe SE, et al. Assessing the relative impact of lupus nephritis on mortality in a population-based systemic lupus erythematosus cohort. Lupus. 2019;28(7):818-825.

[2] Austermann J, et al. S100 proteins in rheumatic diseases. Nat Rev Rheumatol. 2018;14(9):528-541.
[3] Tydén $\mathrm{H}$, et al. Pro-inflammatory $\mathrm{S} 100$ proteins are associated with glomerulonephritis and anti-dsDNA antibodies in systemic lupus erythematosus. Lupus. 2017;26(2):139-149.

[4] Yee CS, et al. Numerical scoring for the BILAG-2004 index. Rheumatology. 2010; 49(9):1665-1669.

[5] McCarthy et al. Short-term efficacy and safety of rituximab therapy in refractory systemic lupus erythematosus: Results from the british isles lupus assessment group biologics register. Rheumatol. 2018;57(3):470-479.

Acknowledgments: Clinical information and serum/urine samples from SLE patients were provided by BILAG-BR centres.

Disclosure of Interests: Jennifer Davies: None declared, Angela Midgley: None declared, Sean Donohue: None declared, Ian N. Bruce Grant/research support from: Genzyme Sanofi, GSK, and UCB, Consultant of: Eli Lilly, AstraZeneca, UCB, Iltoo, and Merck Serono, Speakers bureau: UCB, Michael Beresford: None declared, Christian Hedrich Grant/research support from: Research grant support from Novartis (Molecular pathophysiology of psoriasis)., Speakers bureau: Speaker honoraria from Roche (pathophysiology of polyarticular JIA and systemic JIA); involved in advisory boards for Novartis (systemic JIA and IL-1 mediated diseases).

DOI: 10.1136/annrheumdis-2020-eular.1180

\section{THU0251 \\ IMMUNOPHENOTYPIC CLUSTERS OF SLE PATIENTS REVEAL SUBGROUPS WITH SEVERE DISEASE RESISTANT TO CONVENTIONAL THERAPY}

A. Psarras $^{1}$, D. Li ${ }^{2}$, A. Alase ${ }^{1}$, Z. Wigston ${ }^{1}$, I. Bruce ${ }^{3}$, N. Mchugh ${ }^{2}$, E. Vital $^{1}$ ${ }^{1}$ University of Leeds, Leeds Institute of Rheumatic and Musculoskeletal Medicine, Leeds, United Kingdom; ${ }^{2}$ University of Bath, Bath, United Kingdom; ${ }^{3}$ University of Manchester, Manchester, United Kingdom

Background: Biomarkers to predict response to rituximab include plasmablasts and, in the current MASTERPLANS consortium, Sm/U1RNP antibodies and high expression of IFN Score B (a subset of interferon-stimulated genes that predict more clinical outcomes than a classic interferon signature). The relationships amongst these biomarkers and their association with response to conventional therapies are less well described

Objectives: To analyse the inter-relationships amongst immune biomarkers in two independent SLE cohorts in association with disease activity and stage of therapeutic pathway.

Methods: CONVAS is a cohort of unselected SLE patients; data available include current and historic disease activity, use of biologic therapy, flow cytometry, gene expression (IFN Score A and IFN Score B), and immunoprecipitation for autoantibodies $(n=91)$. BILAG-BR is a British registry study for SLE patients commencing biologics; data available include current and historic disease activity, gene expression (IFN Score A and IFN Score B) and immunoprecipitation for autoantibodies $(n=112)$. In both cohorts, biologics were only prescribed to patients with active disease (BILAG $1 \times$ A or $2 \times \mathrm{B}$ ) and failure of either cyclophosphamide or mycophenolate. Given the mixture of continuous and categorical variables, data were clustered using Gower distance and Partitioning Around Medioids. K was chosen using silhouette coefficient and clusters visualised with t-Distributed Stochastic Neighbor Embedding (t-SNE).

Results: There were 6 clusters. In rituximab-naïve patients:

1. Sm/U1RNP+, Ro60-, highest IFN Score A, low CD4 ${ }^{+} \mathrm{T}$ cells, low NK cells, high plasmablasts

2. Sm/U1RNP-, Ro60+, medium IFN Score A, low $\mathrm{CD}^{+} \mathrm{T}$ cells, high NK cells, high plasmablasts

3. Sm/U1RNP-, Ro60-, lowest IFN Score A, high CD4+ $\mathrm{T}$ cells, low NK cells, low plasmablasts

Other antibody subtypes and flow cytometric markers did not improve the accuracy of clustering. In rituximab-treated patients, 3 equivalent clusters for antibody subtypes and IFN Score A were observed but differentiated due to flow cytometry findings, as expected after rituximab treatment. Overall, the patients in the cluster defined by Sm/U1RNP antibodies and high IFN Score A were notable for a higher rate of prior disease activity in the renal, neurological and general BILAG domains (Table 1).

Table 1 : Clinical features in unselected SLE patients (CONVAS)

\begin{tabular}{lccc}
\hline System affected (ever) & $\begin{array}{c}\text { Sm/U1RNP \& high IFN } \\
\text { Score A }(\mathbf{n}=\mathbf{2 7})\end{array}$ & $\begin{array}{c}\text { Other } \\
(\mathbf{n = 9 2 )}\end{array}$ & $p$ value \\
\hline General & $14 / 27(52 \%)$ & $24 / 92(26 \%)$ & 0.02 \\
Mucocutaneous & $23 / 27(85 \%)$ & $73 / 92(79 \%)$ & 0.50 \\
Neuro & $10 / 27(37 \%)$ & $17 / 92(19 \%)$ & 0.04 \\
MSK & $25 / 27(93 \%)$ & $83 / 92(90 \%)$ & 0.71 \\
Cardiorespiratory & $9 / 27(33 \%)$ & $20 / 92(22 \%)$ & 0.22 \\
Renal & $12 / 27(44 \%)$ & $15 / 92(16 \%)$ & 0.005 \\
Haematology & $25 / 27(93 \%)$ & $67 / 92(73 \%)$ & 0.03 \\
\hline
\end{tabular}


Analysis of autoantibody status and interferon scores only in BILAG-BR confirmed similar clustering. Across both cohorts, the prevalence of the Sm/U1RNP and high IFN Score A cluster was associated with inadequate response to conventional immunosuppressive treatment (Table 2).

Table 2 : Prevalence according to stage of therapy

\begin{tabular}{|c|c|c|c|}
\hline Treatment group & $\begin{array}{l}\text { Sm/U1RNP \& high } \\
\text { IFN Score A }\end{array}$ & Other & $\begin{array}{l}p \\
\text { value }\end{array}$ \\
\hline $\begin{array}{l}\text { Antimalarial or conventional IS-treated } \\
\text { (CONVAS) }(n=90)\end{array}$ & $16 / 90(17.8 \%)$ & $74 / 90(82 \%)$ & 0.02 \\
\hline $\begin{array}{l}\text { Conventional IS inadequate response, } \\
\text { Previous rituximab (CONVAS) }(n=38)\end{array}$ & $14 / 38(36.8 \%)$ & $24 / 38(63.2 \%)$ & \\
\hline $\begin{array}{l}\text { Conventional IS inadequate response, } \\
\text { starting rituximab (BILAG-BR) }(n=163)\end{array}$ & $51 / 163(31.2 \%)$ & $112 / 163(68.7 \%)$ & $\mathrm{N} / \mathrm{A}$ \\
\hline
\end{tabular}

Conclusion: A cluster of $23 \%$ of unselected SLE patients had more severe immune abnormalities, more severe clinical disease activity and were less likely to be maintained on conventional therapies, with twice as many requiring biologic therapy. Other data in MASTERPLANS have demonstrated that Sm/U1RNP antibodies and IFN Scores predict better response to rituximab. This subgroup of patients may therefore be more appropriate for first-line biologic therapy.

Disclosure of Interests: Antonios Psarras: None declared, Danyang Li: None declared, Adewonuola Alase: None declared, Zoe Wigston: None declared, Ian Bruce Grant/research support from: Genzyme, Sanofi, GSK, UCB, Consultant of: Eli Lilly, AstraZeneca, Iltoo, Merck Serono, Neil McHugh: None declared, Edward Vital Grant/research support from: AstraZeneca, Roche/Genentech, and Sandoz, Consultant of: AstraZeneca, GSK, Roche/Genentech, and Sandoz, Speakers bureau: Becton Dickinson and GSK

DOI: 10.1136/annrheumdis-2020-eular.4351

\section{THU0252 CORRELATION BETWEEN DISEASE ACTIVITY AND MENTAL HEALTH IN SLE PATIENTS: A CROSS- SECTION STUDY WITH SELF-ASSESSMENTS BASED ON SMART SYSTEM OF DISEASE MANAGEMENT (SSDM) MOBILE TOOLS}

Y. Wang ${ }^{1}$, H. Wei ${ }^{2}$, R. Wu ${ }^{3}$, J. Wu ${ }^{4}$, S. Zhang ${ }^{5}$, Q. Li ${ }^{6}, Y . \mathrm{Li}^{7}, \mathrm{Y} . \mathrm{Zhao}^{8}$,

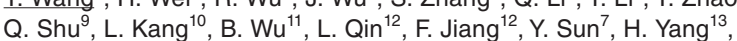
J. Zhang ${ }^{14}, \mathrm{H}$. Xiao ${ }^{15}$, B. Wu ${ }^{15}$, Y. Jia ${ }^{15}, \mathrm{~F} . \mathrm{XiaO}^{15}$, L. Sun ${ }^{16}$ on behalf of SSDM Collaboration Group, China. ${ }^{1}$ The First Affiliated Hospital of BaoTou Medical College, BaoTou, China; ${ }^{2}$ Northern Jiangsu People's Hospital, Jiangsu, China; ${ }^{3}$ The First Affiliated Hospital of Nanchang University, Nanchang, China; ${ }^{4}$ Dazhou Central Hospital, Sichuan, China; ${ }^{5}$ Tongji Hospital Affiliated Tongji Medical College of Huazhong University of Science and Technology, Wuhan, China; ${ }^{6}$ The First People's Hospital of Yunnan Province, Kunming, China: ${ }^{7}$ The Second Affiliated Hospital of Harbin Medical University, Harbin, China; ${ }^{8}$ First Affiliated Hospital of Harbin Medical University, Harbin, China; ${ }^{9}$ Qilu Hospital of Shandong University, Jinan, China; ${ }^{10}$ The First Affiliated Hospital of BaoTou Medical College, Neimenggu, Chile; ${ }^{11}$ The First People's Hospital of Jingzhou, Hubei, China; ${ }^{12}$ The No.3 People's Hospital of HuZhou City, Huzhou, China; ${ }^{13}$ Wuhan No. 1 Hospital, Wuhan, China; ${ }^{14}$ Xi'an No. 5 Hospital, Xi'an, China; ${ }^{15}$ Shanghai Gothic Internet Technology Co., Ltd., Shanghai, China; ${ }^{16}$ Nanjing Drum Tower Hospital, The Affiliated Hospital of Nanjing University Medical School, Nanjing, China

Background: WHO survey showed that the prevalence of anxiety and depression in Chinese population and Chinese patients with chronic diseases were between $3.1 \%-4.2 \%$ and $3.1 \%-7.3 \%$, respectively. SLEDAI-2K and Hospital Anxiety and Depression Scale (HADS) are commonly used to evaluate SLE patients' disease activity and mental health. All the Assessments were mainly performed by health professionals (HCPs) with paper questionnaire previously. SSDM is a novel smart disease management tool that allows patients to do self-assessments on SLEDAI-2K and HADS by mobile App.

Objectives: To investigate the prevalence of anxiety and depression in Chinese patients with SLE and to analyze the potential association between disease activity of SLE and mental health.

Methods: Under the guidance and training by HCPs, SLE patients downloaded SSDM and performed self-assessments bundle of SLEDAI-2K and HADS with SSDM. SLEDAI-2K $<=4,5-9,10-14$ and $>=15$ are defined SLE inactive, low (LDA), moderate (MDA) and high (HDA) disease activity, respectively. SLEDAI-2K score $<=4$ is set as the main criteria for Lupus Low Disease Activity State (LLDAS) and achievement of T2T. HADS score $>=8$ can be diagnosed with anxiety or depression.

Results: From June 2016 to Jan 2020, 3,332 SLE patients (199 male, 3,133 female) with a mean age of $36.34 \pm 12.80(10-91)$ years and the median disease duration of 3.43 years from 216 hospitals performed bundle self-assessments for 4,967 times in total. According to the HADS and SLEDAI-2K Assessment results, the prevalence of anxiety and depression in all patients was $36.7 \%$ and $39.3 \%$ respectively, which was significantly higher than that in the WHO survey in Chinese population and chronic disease patients. The proportion of patients achieved and failed on LLDAS was $53 \%$ and $47 \%$, respectively. The prevalence of anxiety (A) and depression (D) was $19 \%$ and $27 \%$ among LLDAS achievers; $41 \%$ and $47 \%$ among LLDAS failures, respectively $(\mathrm{pA}<0.01, \mathrm{pD}<0.01)$.

According to SLEDAI-2K, in LLDAS, LDA, MDA and HDA subgroups, the prevalence of anxiety and depression was $19 \%, 30 \%, 37 \%, 54 \%$ and $27 \%, 36 \%$, $44 \%, 61 \%$, respectively. The correlation coefficients of anxiety (A) and depression (D) with SLEDAI-2K were $r A=0.9957$ and $r D=0.9819$. It suggested that with the increase of disease activity, the proportion of SLE patients with anxiety and depression increased significantly. (Figure 1)

Conclusion: Conclusion: Higher prevalence of anxiety and depression were Associated with higher levels of disease activity in SLE patients. SSDM is an effective mobile interface to monitor and study entanglement of disease activity and mental health in SLE patients, which build a foundation for proactive interventions physically and mentally in future.

\section{References:}

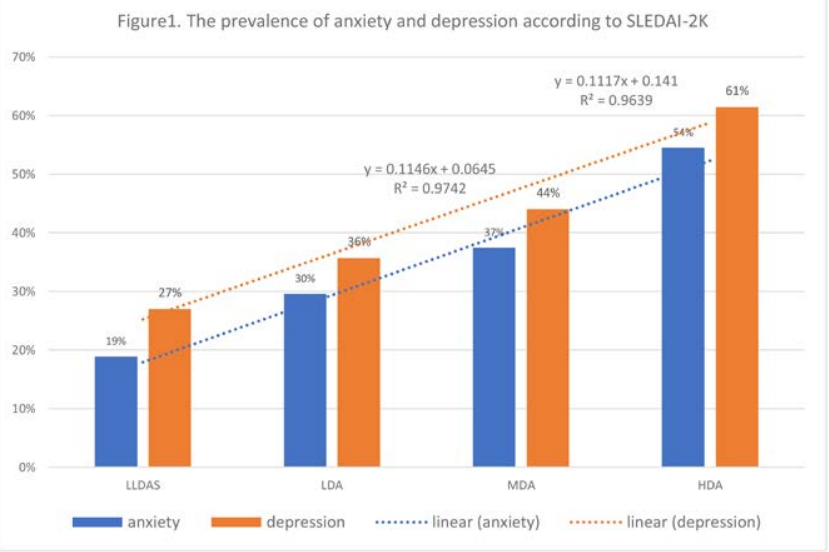

Acknowledgments: SSDM was developed by Shanghai Gothic Internet Technology Co., Ltd.

Disclosure of Interests: None declared

DOI: 10.1136/annrheumdis-2020-eular.1803

\begin{tabular}{|l|l}
\hline THU0253 & FATIGUE AND PAIN REMAIN PROMINENT AND \\
IMPACTFUL IN PATIENTS WITH SYSTEMIC LUPUS \\
ERYTHEMATOSUS (SLE): A CROSS-SECTIONAL \\
SURVEY OF SLE PATIENTS IN THE UNITED STATES
\end{tabular}

J. Birt' ${ }^{1}$ M. Hadi ${ }^{2}$, N. Sargalo², E. Brookes ${ }^{2}$, P. Swinburn ${ }^{2}$, L. Hanrahan ${ }^{3}$, K. Tse ${ }^{3}$, N. Bello Vega ${ }^{4}$, K. Griffing ${ }^{1}$, M. Silk ${ }^{1}$, L. Delbecque ${ }^{5}$, D. L. Kamen ${ }^{6}$. ${ }^{1}$ Indianapolis, Indianapolis, United States of America; ${ }^{2}$ London, London, United Kingdom; ${ }^{3}$ Washington, Washington, United States of America; ${ }^{4}$ Madrid, Madrid, Spain; ${ }^{5}$ Brussels, Brussels, Belgium; ${ }^{6}$ Charleston, Charleston, United States of America

Background: Systemic lupus erythematosus (SLE) is a chronic autoimmune inflammatory condition impacting multiple organ systems. ${ }^{1,2}$ SLE affects approximately 1.5 million Americans, disproportionately females of reproductive age, and is more prevalent in non-Caucasian populations. ${ }^{3}$ Fatigue and pain are some of the most prominent symptoms of SLE, contributing to the heavy disease burden and disruption to daily life. ${ }^{4}$ This study aimed to further understand the burden of SLE. Lilly worked with the Lupus Foundation of America (LFA) and Evidera to develop the SLE-UPDATE (Understanding Preferences, Disease Activity and Treatment Expectations) survey.

Objectives: To understand the patient-perceived symptom burden of SLE, in particular pain and fatigue, within the current landscape of therapeutic options. This study also focused on current treatment patterns in SLE patients.

Methods: This was a cross-sectional, non-interventional, online survey study conducted in partnership with the LFA. English-speaking United States patients aged $\geq 18$ years with a self-reported diagnosis of SLE completed the survey following online screening and informed consent. Descriptive data are presented by means (standard deviation [SD]) for continuous measures, and frequency ( $\mathrm{n}$, $\%)$ for dichotomous measures. Demographic, clinical, and patient-reported outcomes were collected including the FACIT-Fatigue (range 0-52, higher scores 